\title{
On the commutativity of near-rings III
}

\section{Steve Ligh}

Part of the recent work on near-rings has been concerned with sufficient conditions for near-rings to be commutative. Recently Howard $E$. Bell proved that if a d.g. near-ring $R$ has an identity and for each $x, y$ in $R$, there exists an $n(x, y)>1$, such that $(x y-y x)^{n(x, y)}=x y-y x$, then $R$ is a commutative ring. In this paper we drop the requirement that $R$ has an identity and show that the other condition is sufficient (and necessary) for $R$ to be commutative. The inspiration for an important lemma comes from a result of B.H. Neumann.

\section{Introduction}

Part of the recent work on near-rings has been concerned with sufficient conditions for near-rings to be commutative (see [1], [2], [6], [10]). Recently Bell [2] showed that if $R$ is a distributively generated (d.B.) near-ring with an identity, and if for each $x, y$ in $R$, there exists $n>1$, depending on $x$ and $y$, such that $(x y-y x)^{n}=x y-y x$, then $R$ is a commutative ring. He also pointed out that the conclusion will not hold if one drops the existence of an identity. One of the purposes of this paper is to examine the consequence of dropping the hypothesis that $R$ has an identity. It will be shown that $R$ turns out to be a commutative near-ring. Hence we have the following necessary and sufficient condition for a d.g. near-ring to be commutative: A d.g. near-ring $R$ is commutative if and only if for each $x, y$ in $R$, there,

Received 8 February 1972. 
exists $n>1$, depending on $x$ and $y$, such that $(x y-y x)^{n}=x y-y x$. It was shown in [10] that a commutative (distributive) near-ring with 1 is a ring. We see that Bell's result follows from our theorem and the well-known result in ring theory. Herstein proved in [5], as a generalization of the Wedderburn Theorem, that a finite ring is commutative if and only if each divisor of zero is central. Employing this result we shall also show that a finite $\mathrm{d} . \mathrm{g}$. near-ring is commutative if and only if each zero divisor is central.

\section{Preliminaries}

Our definitions are as in [2]. A near-ring ideal $P$ will be called completely prime if $a b \in P$ implies $a \in P$ or $b \in P$. An element $a$ of the near-ring $R$ will be called central if $x a=a x$ for all $x$ in $R$. A near-ring $R$ is called an $N$-system if

(i) $x z=y z$ and $z \neq 0$ implies $x=y$,

(ii) there exists $e \neq 0$ in $R$ such that $e^{2}=e$, and

(iii) there exists $h$ in $R$ such that $h+h=e$.

For easy reference we state the following beautiful result of Neumann [13].

THEOREM 1 (Neumann). The additive group of an N-system is abelian.

$N$-systems are generalizations of near-fields. For an example of an $N$-system that is not a near-field, see [12].

The following lemmas are needed for the proof of Theorem 2 .

LEMMA 1. Let $R$ be a near-ring with no nonzero nilpotent elements. Then $R$ contains a family of completely prime ideals with trivial intersection.

LEMMA 2. Let $R$ be a d.g. near-ring such that for each $x, y$ in $R$, there exists $n>1$, depending on $x$ and $y$, such that $(x y-y x)^{n}=x y-y x$. Then the set of nilpotent elements is an ideal of $R$.

The proof of Lemma 1 is in [1] and Lemma 2 can be found in [2].

LEMMA 3 [4]. Let $R$ be a d.g. near-ring such that $(R,+)$ is abelian. Then $R$ is a ring. 


\section{Main results}

In this section we will furnish necessary and sufficient conditions for d.g. near-rings and finite near-rings to be commutative. First a lemma is needed.

LEMMA 4. Let $R$ be a d.g. near-ring with no nonzero divisors of zero and for each $x, y$ in $R$, let there be $n>1$, depending on $x$ and $y$, such that $(x y-y x)^{n}=x y-y x$. Then $R$ is commutative.

Proof. If there exist $x$ and $y$ such that $x y-y x \neq 0$, then $(x y-y x)^{n-1}=e$ is a nonzero idempotent. Since $R$ has no nonzero divisors of zero and is d.g. , we see that if $w \neq 0$ is a right distributive element of $R$ and $r$ is arbitrary, then $e(e r-r)=0$ implies $e r=r$ and $(r e-r) w=0$ implies $r e=r$. Hence $e$ is an identity of $R$. In fact, any nonzero idempotent is $e$.

Now we wish to show that $(R,+)$ is abelian by demonstrating that either every element of $(R,+)$ is of order two or $R$ is an $N$-system. Let $x, y$ and $z$ be in $R$ such that $x z=y z$ and $z \neq 0$. If $z$ is central then $z x=z y$ implies $x=y$. If $z$ is not central, then there exists $t \neq 0$ such that $z t-t z \neq 0$. Thus $z(z t-t z) \neq 0$ implies that there exists $m>1$ such that $(z(z t)-(z t) z)^{m-1}=e$. Consequently $z$ has a right inverse $z^{\prime}$. Hence $x z=y z$ implies that $x=x z z^{\prime}=y z z^{\prime}=y$.

Suppose $e+e \neq 0$ and is not central. From above, $e+e$ has a right inverse $h$. Since $R$ has no nonzero divisors of zero, $h$ is also a left inverse. Thus $h(e+e)=e$ implies that he $+h e=e$. Hence $R$ is an $N$-system and $(R,+)$ is abelian by Theorem 1 .

Suppose $e+e \neq 0$ and is central, then $e+e$ is also right distributive. Let $a, b$ be in $R$. Expanding $(a+b)(e+e)$ by using both distributive laws, we get $a+b=b+a$. Again $(R,+)$ is abelian.

If $e+e=0$, then each element of $(R,+)$ is of order two and hence $(R,+)$ is abelian.

Now by Lema $3, R$ is a ring and the proof is complete.

We are now ready to prove the following theorem. 
THEOREM 2. A d.g. near-ring $R$ is commatative if and only if for each $x, y$ in $R$, there exists $n>1$, depending on $x$ and $y$, such that $(x y-y x)^{n}=x y-y x$.

Proof. If every element of $R$ is nilpotent, then clearly $R$ is comutative. If $R$ has no nonzero nilpotent elements, then, by Lemma 1 , there exists a family of completely prime ideals $P$ with trivial intersection. For each $P, R / P$ is d.g. and has no zero divisors and $(x y-y x)^{n}=x y-y x$ for each $x, y$ in $R / P$. Thus by Lemma $4, R / P$ is commutative. Hence for each $a, b$ in $R,(a b-b a)$ is in $P$. Consequently $a b=b a$.

Now let $N$ be the set of nilpotent elements. By Lemma $2, N$ is an ideal of $R$. Now $R / N$ has no nonzero nilpotent elements. By the above argument, $R / N$ is commutative and hence $(a b-b a)$ is in $N$ for each $a, b$ in $R$. Thus $a b=b a$.

This completes the proof.

In [9] it was shown that every element of a commutative (distributive) near-ring $R$ is either a zero divisor or $R$ is a ring. Thus we obtain the following result of Bell.

COROLLARY 1 (Bell). Let $R$ be $a$ d.g. near-ring with 1 and for each $x, y$ in $R$, let there be $n(x, y)>1$, such that $(x y-y x)^{n}=x y-y x$. Then $R$ is a commutative ring.

Herstein proved in [5] that a finite ring is comutative if and only if all the zero divisors are central. Now we prove a similar theorem for finite d.g. near-rings. First we state the following result.

LEMMA 5 [8]. Let $R$ be a finite near-ring with identity 1 such that $(-1) x=x$ implies $x=0$. Then $(R,+)$ is abelian.

THEOREM 3. A finite d.g. near-ring $R$ is commutative if and only if all the zero divisors are central.

Proof. Suppose every zero divisor is central. If every element is a zero divisor, then clearly $R$ is commutative. On the other hand, if $R$ does not have any nonzero divisor of zero, then $R$ is a field [7, Corollary 3]. So we may assume that $M$, the set of elements in $R$ which 
are not divisors of zero, is not empty and properly contained in $R$. It was shown in [11, Theorem 3.2] that $M$ is a multiplicative group whose identity $I$ is also the identity for $R$.

Now consider the map $\alpha:(R,+) \rightarrow(R,+)$ defined by $x \alpha=(-1) x$ for every $x$ in $R$. It is easily shown that $\alpha$ is an automorphism of $(R,+)$ and that $\alpha^{2}=I$. Suppose $x=(-1) x$ for some $x$ in $R$. Then we have the following cases:

Case 1. $x \neq 0$ and $x$ is not a zero divisor. Since $x$ is not a zero divisor there exists a $y$ in $R$ such that $x y=1$. Thus $1=x y=(-1) x y=-1$ and $1+1=0$. So we have that every element in $(R,+)$ is of order two and hence $(R,+)$ is abelian. By Lemma $3, R$ is a ring and thus commutative.

Case 2. $x \neq 0$ and $x$ is a zero divisor. By assumption $x$ is central and $x=(-1) x$ implies that $x=x(-1)$. Thus $x+x=0$ and $x(1+1)=0$. It follows that $(1+1)$ is central and hence right distributive. Now if $a$ and $b$ are any two elements of $(R,+)$, then expanding $(a+b)(1+1)$ by using both distributive laws we get that $a+b=b+a$. Again $R$ is a ring by Lemma 3 and hence commutative.

Case 3. $x=0$. By Lemma $5,(R,+)$ is abelian and again $R$ is commutative.

This completes the proof.

REMARKS. Theorems 2 and 3 cannot be extended to arbitrary near-rings, for a finite near-field has the property that $(x y-y x)^{n}=x y-y x$ and all the zero divisors are central. Yet there are near-fields which are not commutative. For examples of commutative near-rings which are not rings, see $[3,2.5, \# 36]$.

\section{References}

[1] Howard E. Bell, "Near-rings in which each element is a power of itself", Bull. Austral. Math. Soc. 2 (1970), 363-368.

[2] Howard E. Bell, "Certain near-rings are rings", J. London Math. Soc. (2) 4 (1971), 264-270. 
[3] James R. Clay, "The near-rings on groups of low order", Math. 2. 104 (1968), 364-371.

[4] A. Fröhlich, "Distributively generated near-rings, (I. Ideal theory)", Proc. London Math. Soc. (3) 8 (1958), 76-94.

[5] I.N. Herstein, "A proof of a conjecture of Vandiver", Proc. Amer. Math. Soc. 1 (1950), 370-371.

[6] Steve Ligh, "On boolean near-rings", Bull. Austral. Math. Soc. 1 (1969), 375-379.

[7] Steve Ligh, "Near-rings with descending chain condition", Compositio Math. 21 (1969), 162-166.

[8] Steve Ligh, "A generalization of a theorem of Zassenhaus", Canad. Math. Bulz. 12 (1969), 677-678.

[9] Steve Ligh, "On the commutativity of near rings", Kyungpook Math. J. 10 (1970), 105-106.

[10] Steve Ligh, "On the commutativity of near rings, II", Kyungpook Math. J. 11 (1971), 159-163.

[11] S. Ligh and J.J. Malone, Jr, "Zero divisors and finite near-rings", J. Austral. Math. Sac. 11 (1970), 374-378.

[12] Steve Ligh, B. McQuarrie and O. Slotterbeck, "On near-fields", J. London Math. Soc. (to appear).

[13] B.H. Neumann, "On the commutativity of addition", J. London Math. Soc. 15 (1940), 203-208.

Department of Mathematics, University of Southwestern Louisiana, Lafayette, Louisiana, USA. 